\title{
Opening the black box: qualitative methods training from the Wolds Primary Care Research Network (WoReN)
}

From its inception, WoReN has endeavoured to meet the demands of its members. Qualitative methods have, finally, been accepted by the mainstream, and are no longer used only by 'consenting eccentrics"! This sea change had been reflected in the research needs of WoReN members. This has resulted in Dr Rosaline Barbour (a medical sociologist from the University of Glasgow) and Valerie Featherstone (research facilitator for the network) entering into a long-term collaboration to design and run innovative, extended, workshop formats. These workshops aim to unlock the black box of qualitative research methods, including data analysis.

A shortened version of these workshops was accepted and run at last year's North American Primary Care Research Group's Twenty-Eighth Annual Meeting, held in Florida in November 2000 - an indication of the international acceptance of qualitative methods and of the Barbour/ WoReN variety in particular.

Dr Barbour has over 20 years' experience of qualitative research, and has been designing and running workshops for the last five years. The WoReN workshops reflect this expertise and present qualitative research as a 'craft skill' located midway between the extremes of esoteric and mechanistic approaches. The first workshop consists of a consideration of the elements which underpin qualitative research methods, including theoretical issues, sampling and research design. In addition, the practicalities of conducting interviews and running focus groups are discussed, and participants actually generate their own data set at the workshop, using focus groups. The second workshop involves participants in interrogating and ana- lysing this data. These workshops run over two separate days, with a gap in between to allow tapes to be fully transcribed, in order to give participants an experience as close to actual research practice as possible. As the workshops themselves have evolved over time, a 'virtual data set' has accumulated, which gives each new set of participants a chance to engage first-hand with the constant comparative method of analysis. Thus they can contextualize themes and insights arising in their own workshops with reference to our 'virtual project'. The impact of issues such as gender, age, professional/lay views, socioeconomic circumstances or any other factors which emerge as having a bearing on the content of generated data can then be considered. To derive maximum benefit from these workshops, participants need to attend both days.

The next workshop, to be held closer to home, has been funded by the Northern and Yorkshire Regional R\&D Training Bursary Scheme. This is therefore offered free of charge to all those who wish to attend. It will be tailored to reflect the interests and needs of participants, and will be held in or near Hull, East Yorkshire, on 23 and 27 March 2001. If you are interested in this workshop, please contact Val Featherstone at WoReN to discuss your particular experience and research training needs.

WoReN, College House, East Riding Campus, Beverley Road, Willerby HU10 6NS.

Tel/Fax: 01482 466958. Email: v.featherstone@ medschool.hull.ac.uk 